\title{
Numerical Solution of Gardner Equation Via Composite Finite Difference Scheme
}

\author{
S. A. El -Morsy a,b \\ ${ }^{a}$ Basic Science Department, Nile Higher Institute for Engineering and Technology, Mansoura, Egypt. \\ ${ }^{\mathrm{b}}$ Current address: Department of Mathematics, College of Science and Arts in Al-Badaya, Qassim \\ University, Al-Badaya, Al-Qassim Province, Saudi Arabia. \\ dr.salwaabdo@nilehi.edu.eg
}

\begin{abstract}
:
In this paper, a new numerical solution of Gardner equation via composite finite difference scheme is introduced. Numerical experiments compare the approximate solution and exact solution.
\end{abstract}

Keywords: Evolution equations; Gardner equation; numerical solution of partial differential equations; composite finite difference scheme.

\section{Introduction}

Partial differential equations play a very important role in various scientific and engineering fields. Such as fluid mechanics, plasma physics, optical fibers, solid state physics and geochemistry. In recent years a variety of powerful and efficient methods have been proposed. For example, SineCosine method [1], Exp-function method [2], the Darboux transformation [3], the Lie group analysis [4], the modified homogeneous balance method [5], and the extended tanh method [6]. New methods for determining solutions of partial differential equations can be given in [7-15]. The Gardner equation belongs to the category of integrable non-linear partial differential equations. The introduction of this equation is attributed to the famous mathematician Clifford Gardner in 1968 [16]. It is an important model to understand the propagation of negative ion acoustic plasma waves [17]. We can derive Gardner equation from the system of plasma motion equations in one dimension with arbitrarily charged cold. The Gardner equation is a good model for describing internal waves with large amplitudes [18]. In [19] The tanh method is applied for generating interacting solutions for this equation. Some interacting of two wave solutions were presented in [20]. These solutions have various terms including trigonometric or hyperbolic functions in rational forms. $\mathrm{G}^{\prime} / \mathrm{G}$ gave Some solitary wave, periodic, exponential, rational and complex-type traveling wave solutions [21]. Numerical solutions of Gardner equations are 
presented in various papers. The conservative finite difference schemes are developed to determine propagation of one soliton and collusion of two soliton solutions numerically [22]. Restrictive Taylor's technique has been implemented to simulate the propagation of some solutions numerically [23].

Composite finite difference scheme (CFDS) has been introduced and applied to some nonlinear equations, such as Burger equation, $\mathrm{KdV}$ equation, $\mathrm{KdVB}$ equation and reaction diffusion equations [24-25]. In this article, a new numerical solution of Gardner equation via composite finite different scheme is obtained.

\section{Composite Finite Difference Scheme}

Consider the general Gardner equation [26] of the form

$$
\mathcal{U}_{t}+\left(\alpha \mathcal{U}+\beta \mathcal{U}^{2}\right) \mathcal{U}_{x}+\gamma \mathcal{U}_{x x x}=0, \quad(x, t) \in Z_{T}
$$

Where $Z_{T}=\sigma \times \zeta, \sigma=(a, b), \zeta=(0, \mathrm{~T}), a$ and $b$ are real positive constants, $\alpha, \beta$ and $\gamma$ are parameters. We consider equation (1) associated with initial condition $\mathcal{U}(x, 0)=\mathcal{U}_{0}(x)$. In finite difference method [24] the domain is discretized forming a grid of a finite number of intersected points with horizontal step $h=\frac{b-a}{N}$, where $N$ is the number of horizontal intervals, $0<\imath<$ $N$ and $\kappa$ is the time step such that $\mathrm{T}=\kappa j, 0<j<M$.

Equation (1) known as the mixed $\mathrm{KdV}-\mathrm{mKdV}$ equation which have very widely applications in physics, plasma, Quantum Field theory. We can rewrite it in the form

$$
\mathcal{U}_{t}=-\left(\alpha \mathcal{U}+\beta \mathcal{U}^{2}\right) \mathcal{U}_{x}-\gamma \mathcal{U}_{x x x}
$$

Multiply both sides of (2) by $\frac{d F}{d u}$, where $F(\mathcal{U})$ is a continuous and differentiable function, we obtain

$$
\frac{d F}{d \mathcal{U}} \frac{\partial \mathcal{U}}{\partial t}=-F^{\prime}(\mathcal{U})\left(\left(\alpha \mathcal{U}+\beta \mathcal{U}^{2}\right) \mathcal{U}_{x}+\gamma \mathcal{U}_{x x x}\right)
$$

In Equation (3) reset $\mathcal{U} \mathcal{U}_{x}=\frac{1}{2} \mathcal{U}^{2}$, we have

$$
\frac{\partial F}{\partial t}=-F^{\prime}(\mathcal{U})\left((\alpha+\beta \mathcal{U}) \frac{\mathcal{U}_{x}^{2}}{2}+\gamma \mathcal{U}_{x x x}\right)
$$

FDM based on replacing derivatives by difference formulas [30, 31] as follows, for $1 \leq \imath \leq 2$ we apply the forward difference formulas,

$$
\begin{aligned}
& \left(u_{x}\right)_{l}^{j}=\frac{-3 u_{\imath}^{j}+4 \mathcal{u}_{\imath+1}^{j}-\mathcal{u}_{\imath+2}^{j}}{2 h}, \\
& \left(\mathcal{U}_{x x x}\right)_{i}^{j}=\frac{-5 \mathcal{U}_{\imath}^{j}+18 \mathcal{U}_{\imath+1}^{j}-24 \mathcal{U}_{l+2}^{j}+14 \mathcal{U}_{\imath+3}^{j}-3 \mathcal{U}_{\imath+4}^{j}}{2 h^{3}}, \\
& \left(\mathcal{U}_{t}\right)_{\imath}^{j}=\frac{\mathcal{U}_{\imath}^{j+1}-\mathcal{U}_{\iota}^{j}}{\kappa}, \quad\left(F_{t}\right)_{\imath}^{j}=\frac{F\left(\mathcal{U}_{\imath}^{j+1}\right)-F\left(\mathcal{U}_{\imath}^{j}\right)}{\kappa} .
\end{aligned}
$$

Substitute from (5) into (4) we get, 


$$
\begin{aligned}
F\left(\mathcal{U}_{i}^{j+1}\right)=F( & \left.\mathcal{U}_{\imath}^{j}\right) \\
& -\kappa F^{\prime}\left(\mathcal{U}_{\imath}^{j}\right)\left(\left(\alpha+\beta \mathcal{U}_{\imath}^{j}\right) \frac{-3\left(\mathcal{U}_{\imath}^{j}\right)^{2}+4\left(\mathcal{U}_{\imath+1}^{j}\right)^{2}-\left(\mathcal{U}_{\imath+2}^{j}\right)^{2}}{4 h}\right. \\
& \left.+\frac{-5 \mathcal{U}_{\imath}^{j}+18 \mathcal{U}_{\imath+1}^{j}-24 \mathcal{U}_{\imath+2}^{j}+14 \mathcal{U}_{\imath+3}^{j}-3 \mathcal{U}_{\imath+4}^{j}}{2 h^{3}}\right) .
\end{aligned}
$$

When $3 \leq 1 \leq \mathrm{N}-2$ we apply the central difference formulas,

$$
\begin{aligned}
& \left(u_{x}\right)_{l}^{j}=\frac{u_{l+1}^{j}-u_{l-1}^{j}}{2 h}, \\
& \left(u_{x x x}\right)_{l}^{j}=\frac{u_{l+2}^{j}-2 u_{l+1}^{j}+2 u_{l-1}^{j}-u_{l-2}^{j}}{2 h^{3}}
\end{aligned}
$$

From (7) substitute in (4), we obtain

$$
\begin{aligned}
& F\left(\mathcal{U}_{i}^{j+1}\right)=F\left(\mathcal{U}_{\imath}^{j}\right) \\
& -\kappa F^{\prime}\left(\mathcal{U}_{\imath}^{j}\right)\left(\left(\alpha+\beta \mathcal{U}_{\imath}^{j}\right) \frac{\left(\mathcal{U}_{l+1}^{j}\right)^{2}-\left(\mathcal{U}_{\imath}^{j}\right)^{2}}{4 h}\right. \\
& \left.+\frac{u_{l+2}^{j}-2 u_{l+1}^{j}+2 u_{l-1}^{j}-u_{l-2}^{j}}{2 h^{3}}\right) .
\end{aligned}
$$

When $N-1 \leq \imath \leq N$ we apply the backward difference formulas,

$$
\begin{aligned}
& \left(\mathcal{U}_{x}\right)_{l}^{j}=\frac{3 U_{l}^{j}-4 \mathcal{U}_{l-1}^{j}+U_{l-2}^{j}}{2 h}, \\
& \left(u_{x x x}\right)_{l}^{j}=\frac{5 u_{l}^{j}-18 U_{l-1}^{j}+24 \mathcal{U}_{l-2}^{j}-14 \mathcal{U}_{l-3}^{j}+3 u_{l-4}^{j}}{2 h^{3}},
\end{aligned}
$$

Substitute from (9) into (4) we get

$$
\begin{aligned}
& F\left(\mathcal{U}_{i}^{j+1}\right)=F\left(\mathcal{U}_{\imath}^{j}\right) \\
& -\kappa F^{\prime}\left(\mathcal{U}_{\imath}^{j}\right)\left(\left(\alpha+\beta \mathcal{U}_{\imath}^{j}\right) \frac{3\left(\mathcal{U}_{\imath}^{j}\right)^{2}-4\left(\mathcal{U}_{\imath-1}^{j}\right)^{2}+\left(\mathcal{U}_{\imath-2}^{j}\right)^{2}}{4 h}\right. \\
& \left.+\frac{5 u_{l}^{j}-18 u_{l-1}^{j}+24 u_{l-2}^{j}-14 u_{l-3}^{j}+3 u_{l-4}^{j}}{2 h^{3}}\right) \text {. }
\end{aligned}
$$

\subsection{Exponential Finite Difference Method}

In this sub section we apply the exponential finite difference method (Exp FDM) which was developed by Bhattachary [27, 28]. He used Exp FDM for solving the one-dimensional heat 
conduction in a solid slab. In [29] aA. R. Bahadir applied Exp FDM to find the numerical solution of Korteweg de Vries equation. In [24] M. S. EL-AZAB et al studied the stability of CFDS when applied to linearized version of $\mathrm{KdVB}$ equation.

In Exp FDM, we set $F(\mathcal{U})=\operatorname{Ln} \mathcal{U}$, then $F^{\prime}(\mathcal{U})=\frac{1}{u}$ substitute in equations (6), (8) and (10) we obtain, for $1 \leq 1 \leq 2$

$$
\begin{aligned}
\operatorname{Ln}\left(\mathcal{U}_{i}^{j+1}\right)= & \operatorname{Ln}\left(\mathcal{U}_{\imath}^{j}\right) \\
& -\frac{\kappa}{\mathcal{U}_{\imath}^{j}}\left(\left(\alpha+\beta \mathcal{U}_{\imath}^{j}\right) \frac{-3\left(\mathcal{U}_{\imath}^{j}\right)^{2}+4\left(\mathcal{U}_{\imath+1}^{j}\right)^{2}-\left(\mathcal{U}_{\imath+2}^{j}\right)^{2}}{4 h}\right. \\
& \left.+\frac{-5 \mathcal{U}_{\imath}^{j}+18 \mathcal{U}_{\imath+1}^{j}-24 \mathcal{U}_{\imath+2}^{j}+14 \mathcal{U}_{\imath+3}^{j}-3 \mathcal{U}_{\imath+4}^{j}}{2 h^{3}}\right),
\end{aligned}
$$

When $3 \leq 1 \leq N-2$, equation (8) becomes,

$$
\begin{aligned}
& \operatorname{Ln}\left(\mathcal{U}_{i}^{j+1}\right)=\operatorname{Ln}\left(\mathcal{U}_{\imath}^{j}\right) \\
& -\frac{\kappa}{\mathcal{U}_{\imath}^{j}}\left(\left(\alpha+\beta \mathcal{U}_{\imath}^{j}\right) \frac{\left(\mathcal{U}_{\imath+1}^{j}\right)^{2}-\left(\mathcal{U}_{\imath}^{j}\right)^{2}}{4 h}\right. \\
& \left.+\frac{\mathcal{u}_{\imath+2}^{j}-2 \mathcal{U}_{\imath+1}^{j}+2 \mathcal{u}_{l-1}^{j}-\mathcal{U}_{\imath-2}^{j}}{2 h^{3}}\right) \\
& \left(\operatorname{Ln}\left(\mathcal{U}_{i}^{j+1}\right)=\operatorname{Ln}\left(\mathcal{U}_{\imath}^{j}\right)\right. \\
& -\frac{\kappa}{\mathcal{U}_{\iota}^{j}}\left(\left(\alpha+\beta \mathcal{U}_{\iota}^{j}\right) \frac{3\left(\mathcal{U}_{\imath}^{j}\right)^{2}-4\left(\mathcal{U}_{l-1}^{j}\right)^{2}+\left(\mathcal{U}_{l-2}^{j}\right)^{2}}{4 h}\right. \\
& \left.+\frac{5 \mathcal{U}_{l}^{j}-18 \mathcal{U}_{l-1}^{j}+24 \mathcal{U}_{l-2}^{j}-14 \mathcal{U}_{l-3}^{j}+3 \mathcal{U}_{l-4}^{j}}{2 h^{3}}\right) \text {, } \\
& \mathrm{N}-1 \leq \mathrm{1} \leq \mathrm{N}
\end{aligned}
$$

Simplify Equations (11-13) we obtain the following equations,

$$
\begin{aligned}
u_{\mathrm{i}}^{\mathrm{j}+1}=\mathcal{U}_{1}^{\mathrm{j}} \exp \left(\frac { - \kappa } { \mathcal { u } _ { 1 } ^ { \mathrm { j } } } \left(\left(\alpha+\beta \mathcal{u}_{\imath}^{j}\right) \frac{-3\left(\mathcal{U}_{1}^{\mathrm{j}}\right)^{2}+4\left(\mathcal{u}_{1+1}^{\mathrm{j}}\right)^{2}-\left(\mathcal{u}_{1+2}^{\mathrm{j}}\right)^{2}}{4 \mathrm{~h}}\right.\right. \\
\left.\left.+\frac{-5 \mathcal{u}_{1}^{\mathrm{j}}+18 \mathcal{u}_{1+1}^{\mathrm{j}}-24 \mathcal{u}_{1+2}^{\mathrm{j}}+14 \mathcal{u}_{1+3}^{\mathrm{j}}-3 \mathcal{U}_{1+4}^{\mathrm{j}}}{2 \mathrm{~h}^{3}}\right)\right), 1 \leq 1 \\
\leq 2,
\end{aligned}
$$




$$
\begin{aligned}
& \mathcal{U}_{\mathrm{i}}^{\mathrm{j}+1}=\mathcal{U}_{1}^{\mathrm{j}} \exp \left(\frac { - \kappa } { \mathcal { U } _ { 1 } ^ { \mathrm { j } } } \left(\left(\alpha+\beta \mathcal{U}_{\imath}^{j}\right) \frac{\left(\mathcal{U}_{1+1}^{\mathrm{j}}\right)^{2}-\left(\mathcal{U}_{1}^{\mathrm{j}}\right)^{2}}{2 \mathrm{~h}}\right.\right. \\
& \left.\left.+\frac{u_{1+2}^{j}-2 u_{1+1}^{j}+2 u_{1-1}^{j}-u_{1-2}^{j}}{2 h^{3}}\right)\right) \\
& \mathcal{u}_{\mathrm{i}}^{\mathrm{j}+1}=\mathcal{u}_{1}^{\mathrm{j}} \exp \left(-\frac{\kappa}{\mathcal{u}_{1}^{\mathrm{j}}}\left(\left(\alpha+\beta \mathcal{U}_{\imath}^{j}\right) \frac{3\left(\mathcal{u}_{1}^{\mathrm{j}}\right)^{2}-4\left(\mathcal{U}_{1-1}^{\mathrm{j}}\right)^{2}+\left(\mathcal{u}_{1-2}^{\mathrm{j}}\right)^{2}}{4 \mathrm{~h}}\right.\right. \\
& \left.\left.+\frac{5 \mathcal{U}_{1}^{\mathrm{j}}-18 \mathcal{U}_{1-1}^{\mathrm{j}}+24 \mathcal{u}_{1-2}^{\mathrm{j}}-14 \mathcal{U}_{1-3}^{\mathrm{j}}+3 \mathcal{U}_{1-4}^{\mathrm{j}}}{2 \mathrm{~h}^{3}}\right)\right) \text {, } \\
& \mathrm{N}-1 \leq \mathrm{l} \leq \mathrm{N} .
\end{aligned}
$$

Logarithmic finite difference method was introduced by M. S. El-Azab and S. A. El Morsy for solving the nonlinear evolution equations. They applied the method to a class of solitary waves equations, such as KdV and KdVB equations [30]. In [25] M. S. El-Azab et al applied Logarithmic finite difference method to nonlinear reaction diffusion equation. The method is simple and effective for arbitrarily large values of parameters.

Consider $F(\mathcal{U})=\exp (\mathcal{U})$, then $\mathrm{F}^{\prime}(\mathcal{U})=\exp (\mathcal{U})$, substitute in equations (6), (8) and (10) to obtain,

we obtain,

$$
\begin{aligned}
\exp \left(\mathcal{U}_{i}^{j+1}\right)= & \exp \left(\mathcal{U}_{\imath}^{j}\right) \\
& -\kappa \exp \left(\mathcal{U}_{\imath}^{j}\right)\left(\left(\alpha+\beta \mathcal{U}_{\imath}^{j}\right) \frac{-3\left(\mathcal{U}_{\imath}^{j}\right)^{2}+4\left(\mathcal{U}_{\imath+1}^{j}\right)^{2}-\left(\mathcal{U}_{\imath+2}^{j}\right)^{2}}{4 h}\right. \\
& \left.+\frac{-5 \mathcal{U}_{\imath}^{j}+18 \mathcal{U}_{\imath+1}^{j}-24 \mathcal{U}_{\imath+2}^{j}+14 \mathcal{U}_{\imath+3}^{j}-3 \mathcal{U}_{\imath+4}^{j}}{2 h^{3}}\right), 1 \leq 1 \leq 2
\end{aligned}
$$

When $3 \leq 1 \leq \mathrm{N}-2$, equation (8) becomes,

$$
\begin{aligned}
\exp \left(\mathcal{U}_{i}^{j+1}\right)= & \exp \left(\mathcal{U}_{\imath}^{j}\right) \\
& -\kappa \exp \left(\mathcal{U}_{\imath}^{j}\right)\left(\left(\alpha+\beta \mathcal{U}_{\imath}^{j}\right) \frac{-3\left(\mathcal{U}_{\imath}^{j}\right)^{2}+4\left(\mathcal{U}_{\imath+1}^{j}\right)^{2}-\left(\mathcal{U}_{\imath+2}^{j}\right)^{2}}{4 h}\right. \\
& \left.+\frac{-5 \mathcal{U}_{\imath}^{j}+18 \mathcal{U}_{\imath+1}^{j}-24 \mathcal{U}_{\imath+2}^{j}+14 \mathcal{U}_{\imath+3}^{j}-3 \mathcal{U}_{\imath+4}^{j}}{2 h^{3}}\right)
\end{aligned}
$$




$$
\begin{aligned}
\exp \left(\mathcal{U}_{i}^{j+1}\right)= & \exp \left(\mathcal{U}_{\imath}^{j}\right) \\
& -\kappa \exp \left(\mathcal{U}_{\imath}^{j}\right)\left(\left(\alpha+\beta \mathcal{U}_{\imath}^{j}\right) \frac{3\left(\mathcal{U}_{\imath}^{j}\right)^{2}-4\left(\mathcal{U}_{l-1}^{j}\right)^{2}+\left(\mathcal{U}_{l-2}^{j}\right)^{2}}{2 h}\right. \\
& \left.+\frac{5 \mathcal{U}_{\imath}^{j}-18 \mathcal{U}_{l-1}^{j}+24 \mathcal{U}_{l-2}^{j}-14 \mathcal{U}_{l-3}^{j}+3 \mathcal{U}_{l-4}^{j}}{2 h^{3}}\right), \quad \mathrm{N}-1 \leq \mathrm{l} \leq \mathrm{N}
\end{aligned}
$$

Simplify Equations (17-19) we can obtain the following algebraic system of equations,

$$
\begin{aligned}
u_{i}^{j+1}=\mathcal{U}_{\imath}^{j}+ & \operatorname{Ln}\left(-\kappa\left(\left(\alpha+\beta \mathcal{U}_{\imath}^{j}\right) \frac{-3\left(\mathcal{U}_{\imath}^{j}\right)^{2}+4\left(\mathcal{U}_{\imath+1}^{j}\right)^{2}-\left(\mathcal{U}_{\imath+2}^{j}\right)^{2}}{4 h}\right.\right. \\
& \left.\left.+\frac{-5 \mathcal{U}_{\imath}^{j}+18 \mathcal{U}_{\imath+1}^{j}-24 \mathcal{U}_{\imath+2}^{j}+14 \mathcal{U}_{\imath+3}^{j}-3 \mathcal{U}_{\imath+4}^{j}}{2 h^{3}}\right)+1\right), 1 \leq 1 \leq 2
\end{aligned}
$$

When $3 \leq 1 \leq \mathrm{N}-2$, equation (8) becomes,

$$
\begin{aligned}
& \mathcal{U}_{i}^{j+1}=\mathcal{U}_{\imath}^{j}+\operatorname{Ln}\left(-\kappa\left(\left(\alpha+\beta \mathcal{U}_{\imath}^{j}\right) \frac{-3\left(\mathcal{U}_{\imath}^{j}\right)^{2}+4\left(\mathcal{U}_{\imath+1}^{j}\right)^{2}-\left(\mathcal{U}_{\imath+2}^{j}\right)^{2}}{4 h}\right.\right. \\
& \left.+\frac{-5 \mathcal{U}_{\iota}^{j}+18 \mathcal{u}_{\imath+1}^{j}-24 \mathcal{U}_{\iota+2}^{j}+14 \mathcal{U}_{\iota+3}^{j}-3 \mathcal{U}_{\iota+4}^{j}}{2 h^{3}}\right) \\
& +1) \\
& \mathcal{U}_{i}^{j+1}=\mathcal{U}_{\imath}^{j}+\operatorname{Ln}\left(-\kappa\left(\left(\alpha+\beta \mathcal{U}_{\imath}^{j}\right) \frac{3\left(\mathcal{U}_{\imath}^{j}\right)^{2}-4\left(\mathcal{U}_{l-1}^{j}\right)^{2}+\left(\mathcal{U}_{l-2}^{j}\right)^{2}}{4 h}\right.\right. \\
& \left.\left.+\frac{5 \mathcal{U}_{l}^{j}-18 \mathcal{U}_{l-1}^{j}+24 \mathcal{U}_{l-2}^{j}-14 \mathcal{U}_{l-3}^{j}+3 \mathcal{U}_{l-4}^{j}}{2 h^{3}}\right)+1\right) \text {, } \\
& \mathrm{N}-1 \leq \mathrm{l} \leq \mathrm{N}
\end{aligned}
$$

\section{Numerical Experiments}

\section{Case study 1}

Consider Gardner equation [31] when $\alpha=4, \beta=-3$ and $\gamma=1$, with the initial condition $\mathcal{U}(x, 0)=\frac{2}{12+3 \sqrt{14} \operatorname{Cosh}\left(-\frac{x}{3}+\frac{5}{3}\right)}$ and the exact solution $(x, t)=\frac{2}{12+3 \sqrt{14} \operatorname{Cosh}\left(-\frac{x}{3}+\frac{5}{3}+\frac{t}{27}\right)}$. 
Table 1. Absolute errors for Exp. FDM and Log.FDM when $h=0.1$

\begin{tabular}{|c|l|l|}
\hline \multicolumn{3}{|c|}{$\kappa=0.0001, h=0.1$ and $t=0.001$} \\
\hline $\boldsymbol{x}$ & Exp. FDM & Log. FDM \\
\hline 0.0 & $1.9331 \times 10^{-8}$ & $3.79303 \times 10^{-7}$ \\
\hline 0.1 & $7.2528 \times 10^{-9}$ & $2.80823 \times 10^{-6}$ \\
\hline 0.2 & $2.9034 \times 10^{-9}$ & $3.46015 \times 10^{-6}$ \\
\hline 0.3 & $9.4034 \times 10^{-10}$ & $4.24513 \times 10^{-6}$ \\
\hline 0.4 & $1.8390 \times 10^{-10}$ & $4.96094 \times 10^{-6}$ \\
\hline 0.5 & $2.3134 \times 10^{-10}$ & $5.68259 \times 10^{-6}$ \\
\hline 0.6 & $6.7394 \times 10^{-11}$ & $6.10547 \times 10^{-6}$ \\
\hline 0.7 & $2.0092 \times 10^{-11}$ & $4.4415 \times 10^{-6}$ \\
\hline 0.8 & $4.7687 \times 10^{-11}$ & $4.42334 \times 10^{-6}$ \\
\hline 0.9 & $4.6039 \times 10^{-10}$ & $7.04767 \times 10^{-6}$ \\
\hline 1.0 & $1.4084 \times 10^{-9}$ & $6.42047 \times 10^{-7}$ \\
\hline
\end{tabular}

Table 2. Absolute errors for Exp. FDM and Log. FDM when $h=1.0$

\begin{tabular}{|c|l|c|}
\hline \multicolumn{3}{|c|}{$\kappa=0.0001, h=1$ and $t=0.001$} \\
\hline $\boldsymbol{x}$ & \multicolumn{1}{|c|}{ Exp. FDM } & Log. FDM \\
\hline 0.0 & $3.60921 \times 10^{-7}$ & $1.97198 \times 10^{-6}$ \\
\hline 1.0 & $5.83107 \times 10^{-7}$ & $1.82722 \times 10^{-6}$ \\
\hline 2.0 & $5.431 \times 10^{-7}$ & $1.73770 \times 10^{-6}$ \\
\hline 3.0 & $1.00206 \times 10^{-8}$ & $5.80394 \times 10^{-6}$ \\
\hline 4.0 & $8.86987 \times 10^{-9}$ & $3.45538 \times 10^{-6}$ \\
\hline 5.0 & $4.63718 \times 10^{-12}$ & $1.30074 \times 10^{-6}$ \\
\hline 6.0 & $8.99999 \times 10^{-9}$ & $3.45796 \times 10^{-6}$ \\
\hline 7.0 & $9.94002 \times 10^{-9}$ & $5.80334 \times 10^{-6}$ \\
\hline 8.0 & $2.15564 \times 10^{-9}$ & $6.52653 \times 10^{-6}$ \\
\hline 9.0 & $8.06268 \times 10^{-9}$ & $1.83816 \times 10^{-6}$ \\
\hline 10.0 & $1.41102 \times 10^{-8}$ & $1.97448 \times 10^{-6}$ \\
\hline
\end{tabular}

\section{Case study 2}

Consider Gardner equation [32] when $\alpha=1, \beta=-5$ and $\gamma=1$, with the initial condition $U(x, 0)=0.1\left(1-\tanh \left(\frac{x}{2 \sqrt{30}}\right)\right) \quad$ with exact solution $\quad \mathcal{U}(x, t)=0.1(1-$ $\left.\tanh \left(\frac{x-0.1 t}{2 \sqrt{30}}\right)\right)$ 
Table 3. Absolute errors for Exp. FDM and Log. FDM when $h=0.1$

\begin{tabular}{|l|c|c|}
\hline \multicolumn{3}{|c|}{$\kappa=0.0001, h=0.1$ and $t=0.001$} \\
\hline $\boldsymbol{x}$ & Exp. FDM & Log. FDM \\
\hline 0.0 & $1.53809 \times 10^{-6}$ & $1.63261 \times 10^{-7}$ \\
\hline 0.1 & $1.51488 \times 10^{-6}$ & $4.08396 \times 10^{-7}$ \\
\hline 0.2 & $1.52308 \times 10^{-6}$ & $4.87864 \times 10^{-7}$ \\
\hline 0.3 & $1.51889 \times 10^{-6}$ & $6.30548 \times 10^{-7}$ \\
\hline 0.4 & $1.51844 \times 10^{-6}$ & $7.35689 \times 10^{-7}$ \\
\hline 0.5 & $1.51628 \times 10^{-6}$ & $8.03524 \times 10^{-7}$ \\
\hline 0.6 & $1.51415 \times 10^{-6}$ & $8.35254 \times 10^{-7}$ \\
\hline 0.7 & $1.51148 \times 10^{-6}$ & $6.04582 \times 10^{-7}$ \\
\hline 0.8 & $1.50851 \times 10^{-6}$ & $5.97121 \times 10^{-7}$ \\
\hline 0.9 & $1.50529 \times 10^{-6}$ & $9.42587 \times 10^{-7}$ \\
\hline 1.0 & $1.5017 \times 10^{-6}$ & $1.59377 \times 10^{-7}$ \\
\hline
\end{tabular}

Table 4. Absolute errors for Exp. FDM and Log. FDM when $h=0.1$

\begin{tabular}{|c|l|c|}
\hline \multicolumn{3}{|c|}{$\kappa=0.00001, h=1.0$ and $t=0.0001$} \\
\hline $\boldsymbol{x}$ & \multicolumn{1}{|c|}{ Exp. FDM } & Log. FDM \\
\hline 0.0 & $1.53816 \times 10^{-7}$ & $2.8845 \times 10^{-7}$ \\
\hline 1.0 & $1.51316 \times 10^{-7}$ & $2.96338 \times 10^{-7}$ \\
\hline 2.0 & $1.44889 \times 10^{-7}$ & $3.17768 \times 10^{-7}$ \\
\hline 3.0 & $1.35135 \times 10^{-7}$ & $6.73696 \times 10^{-7}$ \\
\hline 4.0 & $1.23766 \times 10^{-7}$ & $6.16856 \times 10^{-7}$ \\
\hline 5.0 & $1.10903 \times 10^{-7}$ & $5.53123 \times 10^{-7}$ \\
\hline 6.0 & $9.74436 \times 10^{-8}$ & $4.86214 \times 10^{-7}$ \\
\hline 7.0 & $8.41595 \times 10^{-8}$ & $4.20061 \times 10^{-7}$ \\
\hline 8.0 & $7.16318 \times 10^{-8}$ & $3.57718 \times 10^{-7}$ \\
\hline 9.0 & $5.88746 \times 10^{-8}$ & $4.02963 \times 10^{-7}$ \\
\hline 10.0 & $4.88345 \times 10^{-8}$ & $3.84064 \times 10^{-7}$ \\
\hline
\end{tabular}

\section{Conclusion}

Exponential and Logarithmic Finite Difference methods are applied effectively to Gardner equation. Numerical experiments show that the absolute error between numerical solution and exact solution is neglectable for different times and for different exact solutions.

\section{References}

[1] A. M. Wazwaz, The sine-cosine method for obtaining solutions with compact and noncompact structures, Applied Mathematics and Computation, 159 (2) (2004) 559-576.

[2] K. R. Raslan, The Application of He's Exp-Function Method for MKdV and Burgers' Equations with Variable Coefficients. Inter. Journal of Nonlinear Science, 7(2) (2009) 174-81. 
[3] J. Lin, Y.-S. Li, and X. M. Qian, The Darboux transformation of the Schrödinger equation with an energy-dependent potential, Phys. Lett. A 362 (2007) 212-214.

[4] J.J. Duistermaat and J.A.C. Kolk. Lie Groups. Universitext. Springer Verlag, first edition, 2000 .

[5] Liu Chun-Ping, A modified homogeneous balance method and its applications, Commun. Theor. Phys. 56 (2011) 223-227.

[6] A.-M. Wazwaz, The Extended Tanh Method for Abundant Solitary Wave Solutions of Nonlinear Wave Equations, Applied Mathematics and Computation, 187(2) (2007)131-1142.

[7] Abdelsalam, U.M. Allehiany, F.M. Different Nonlinear Solutions of KP Equation in Dusty Plasmas. Arab. J. Sci. Eng. 2018, 43, 399-406.

[8] Baskonus, H.M.; Sulaiman, T.A.; Bulut, H. On the new wave behavior to the Klein-GordonZakharov equations in plasma physics. Indian J. Phys. 2019, 93, 393-399.

[9] G. Łysik, The Borel summable solutions of heat operators on a real analytic manifold, J. Math. Anal. Appl. 410 (2014), 117-123.

[10] H. Tahara, H. Yamazawa, Multisummability of formal solutions to the Cauchy problem for some linear partial differential equations, J. Differential Equations 255 (2013) 3592- 3637.

[11] S. A. El Morsy, M. S. El-Azab and I. L. El-Kalla, $\left(G^{\prime} / G\right)$ and extended $\left(G^{\prime} / G\right)$ methods for solving the nonlinear reaction-diffusion equation and $\mathrm{KdVB}$ equation, Electronic Journal of Mathematical Analysis and Applications (EJMAA),3 (2) (2014), 97-110.

[12] Z. Odibat, S. Momani, Numerical methods for nonlinear partial differential equations of fractional order, Appl. Math. Model., 32 (1) (2008) 28-39.

[13] Hu. H., Tan. M., \& Hu. X., New interaction solutions to the combined KdV-mKdV equation from CTE method, Journal of the Association of Arab Universities for Basic and Applied Sciences, 21(2016) 64-67.

[14] Wei-Feng. Y., Sen-Yue. L., Jun. Y., \& Han-Wei. H., Interactions between Solitons and Cnoidal Periodic Waves of the Gardner Equation, Chinese Physics Letters, 31(7) (2014).

[15] Nishiyama H., \& Noi T. Conservative difference schemes for the numerical solution of the Gardner equation. Computational and Applied Mathematics, 35(1) (2016) 75-95.

[16] Miura RM, Gardner CS, Kruskal MD. Korteweg-de Vries equation and generalizations. II. Existence of conservation laws and constants of motion Journal of Mathematical Physics. 9(1968) 1204-1209.

[17] Das G. C., Ionic thermal effects on ion-acoustic-waves in plasmas with negative-ions, Plasma Phys. Control Fusion, 19(1977) 363-368.

[18] Slyunyaev, A. V., \& Pelinovski, E. N., Dynamics of large-amplitude solitons. Journal of Experimental and Theoretical Physics, 89(1) (1999)., 173-181. 
[19] Jawad, A. J. A. M., New Exact Solutions of Nonlinear Partial Differential Equations Using Tan-Cot Function Method. Studies in Mathematical sciences, 5(2)(2012), 13-25.

[20] Wei-Feng Y., Sen-Yue, L., Jun, Y., \& Han-Wei H., Interactions between Solitons and Cnoidal Periodic Waves of the Gardner Equation. Chinese Physics Letters, 31(7)(2014), 070203.

[21] Naher, H., \& Abdullah F. A., Some new solutions of the combined KdV-MKdV equation by using the improved G/G-expansion method. World Applied Sciences Journal, 16(11)(2012), 1559-1570.

[22] Nishiyama, H., \& Noi, T., Conservative difference schemes for the numerical solution of the Gardner equation. Computational and Applied Mathematics, 35(1) (2016), 75-95.

[23] Rageh, T. M., Salem, G., \& El-Salam, F. A. (2014). Restrictive Taylor Approximation for Gardner and KdV Equations, Int. J. Adv. Appl. Math. and Mech, 1(3)(2014), 1-10.

[24] M. S. EL-Azab, I. L. EL-Kalla and S. A. EL Morsy, Composite finite difference scheme applied to a couple of nonlinear evolution equations, Electronic Journal of Mathematical Analysis and Applications (EJMAA ), 2(2)( 2014), 254-263.

[25] S. A. EL MORSY, Composite finite difference scheme applied to some nonlinear evolution equations, 4(1) (2016), 197-204.

[26] Anwwar Ja'afar Mohamad Jawad, The sine-cosine method for the exact solutions of nonlinear pariail differential equations, IJRRS, 13(1) (2012), 186-191.

[27] M. C. Bhattacharya, A new improved_fnite difference equation for heat transfer during transient change, Appl. Math. Model., 10 (1) (1986), 68-70.

[28] M. C. Bhattacharya, M. G. Davies, The comparative performance of some _fnite difference equations for transient heat conduction problems, Int. J. Numer. Meth. Eng., 21 ( 7) (1987), 1317-1331.

[29] A. R. Bahadir, Exponential finite difference method applied to Kortewge de Vries equation for small times, Appl. Math. and Comp. ,160, 3, (2005), 675- 682.

[30] S. A. El Morsy and M. S. El-Azab, Logarithmic Finite Difference Method Applied to KdVB Equation, American Academic Scholarly Research Journal (AASRJ), 4 ( 2) (2012), 4903-4910.

[31] A. M. Wazwaz, New solitons and kink solutions for the Gardner equation. Communications in nonlinear science and numerical simulation, 12(8) (2007), 1395-1404.

[32] A. M. Wazwaz, Partial Differential Equations and Solitary Waves Theory, Springer-Verlag Berlin Heidelberg, 2009. 